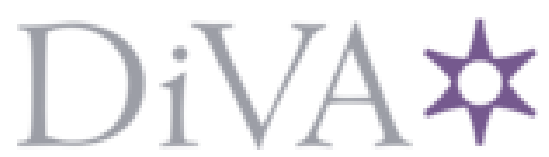

http://www.diva-portal.org

This is the published version of a paper published in The American mathematical monthly.

Citation for the original published paper (version of record):

Raman-Sundström, M. (2015)

A pedagogical history of compactness.

The American mathematical monthly, 122(7): 619-635

http://dx.doi.org/10.4169/amer.math.monthly.122.7.619

Access to the published version may require subscription.

N.B. When citing this work, cite the original published paper.

Copyright 2015 Mathematical Association of America. All Rights Reserved.

Permanent link to this version:

http://urn.kb.se/resolve?urn=urn:nbn:se:umu:diva-106686 


\title{
A Pedagogical History of Compactness
}

\author{
Manya Raman-Sundström*
}

\begin{abstract}
This paper traces the history of compactness from the original motivating questions through the development of the definition to a characterization of compactness in terms of nets and filters. The goal of the article is to clarify the central concepts of open-cover and sequential compactness, including details that a standard textbook treatment tends to leave out.
\end{abstract}

Modern mathematics tends to obliterate history: each new school rewrites the foundations of its subject in its own language, which makes for fine logic but poor pedagogy.

R. Hartshorne

1. WHY STUDY THE HISTORY OF COMPACTNESS? Compactness has come to be one of the most useful notions in advanced mathematics. It can also be seen as a kind of gate-keeper topic. If an undergraduate mathematics student does not understand compactness it is unlikely that he or she will be able to do higher level mathematics. However, for whatever reasons, when we teach compactness and other topics of similar importance, we tend to provide little historical motivation. Students are left more or less on their own to figure out, if they ever do, how definitions and theorems relate to each other and why they take the specific forms they do.

This paper is an attempt to fill in some of the information that the standard textbook treatment of compactness leaves out. It is not a historical article, per se, but a synthesis of historical documents with an eye towards clarifying the main ideas related to compactness. In particular, the paper discusses the origins and development of both open-cover and sequential compactness, how and why open-cover compactness came to be favored, and some modern developments involving nets and filters. We will move fairly slowly and carefully through the early part of the history and a bit more quickly through the more modern developments.

A list of terms related to compactness is given in the Appendix. Since the terms have changed names at various points in history, the list can be useful for keeping the concepts straight. In the main text we will use a combination of historical and modern formulations of the main definitions, lemmas, and theorems, favoring the best ones for readability with originals in footnotes.

2. POSSIBLE MOTIVATIONS FOR COMPACTNESS. Compactness grew out of one of the most productive periods of mathematical activity. In mid to late nineteenth century Europe, advanced mathematics began to take the form we know today. In the background was Cantor's work establishing the beginning of a systematic study of set theory and point-set topology. ${ }^{1}$ Also, many mathematicians-including Weierstrass, Hausdorff, and Dedekind-were worried about the foundations of mathematics and

\footnotetext{
http://dx.doi.org/10.4169/amer.math.monthly.122.7.619

MSC: Primary 01-01

* This paper is based upon my masters thesis [38] at UC Berkeley.

${ }^{1}$ Though Cantor himself turned his interests to transfinite sets, the significance of Cantor's work for topology should be credited to Poincaré. Thanks to Jeremy Gray for this comment.
} 
began to make rigorous many of the ideas that had for centuries been taken for granted. While some of the nineteenth century work can be traced to mathematical concerns of the early Greeks, the level of rigor and abstraction reflects a revolution in mathematical thought.

It is in this context that we will discuss some specific problems that appear to have motivated the concept of compactness. In particular, we will discuss the influence of the study of properties of closed, bounded intervals of real numbers (which will be denoted $[a, b]$ ), spaces of continuous functions, and solutions to differential equations.

Properties of $[\boldsymbol{a}, \boldsymbol{b}]$. In the mid to late nineteenth century, mathematicians began to really understand and specify essential properties of the real line. This work led to two different characterizations of the notion which came to be known as compactness. One characterization, developed by Bolzano and Weierstrass among others, grew out of the study of functions defined on sequences of real numbers. The other characterization, which grew out of work by Heine, Borel, and Lebesgue, was based on topological features, such as the covering of sets by open neighborhoods. We will examine both of these characterizations in more detail.

The origin of sequential compactness is often traced to a theorem, proved rigorously by Weierstrass in 1877, which concerns the behavior of continuous functions defined on closed, bounded intervals of the real line. ${ }^{2}$ The following statement of the theorem comes from Fréchet, who referred to this theorem as a result of Weierstrass.

Theorem 2.1 (Weierstrass). Each function continuous in a limited [equivalent to modern-day "closed and bounded"] interval attains there at least once its maximum. ${ }^{3}$

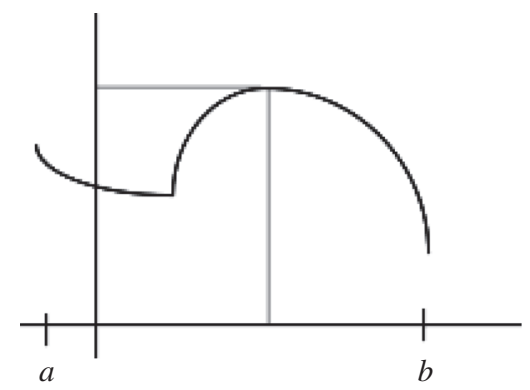

Figure 1. A continuous function on $[a, b]$

Fréchet, who defined sequential compactness in his 1906 thesis, said that his definition came from his desire to generalize this theorem to abstract spaces [43, p. 244]. Weierstrass's theorem owes its essential ideas to Bolzano, who in 1817 working in relative isolation both politically and mathematically in Bohemia, remarkably stated and proved the following:

Lemma 2.2 (Bolzano). If a property $M$ does not apply to all values of a variable quantity $x$, but to all those that are smaller than a certain $u$, there is always a quantity

\footnotetext{
${ }^{2}$ This date refers to one of the earliest publications of the theorem, see [46]. However, it is likely that Weierstrass actually proved it and disseminated it orally, via lectures, perhaps ten years earlier.

${ }^{3}$ From the original French: Weierstrass a en effete démontré que toute fonction continué dans un intervalle limité y atteint au moins une fois son maximum [17, p. 848].
} 
$U$ which is the greatest for those of which it can be asserted that all smaller $x$ possess the property $M .^{4}$

This lemma, today called the least upper bound property for real numbers, was somewhat of a breakthrough in the conceptualization of real numbers. The proof of this lemma provided the first real account of the limiting process, and was used to prove what we now call the intermediate value theorem: if $f$ is continuous on $[a, b]$ with $f(a)<0$ and $f(b)>0$, then for some $x$ between $a$ and $b, f(x)$ will be exactly 0 .

The idea behind Bolzano's proof of the lemma was to use interval bisection, that is to narrow in on the least upper bound by throwing away points of the set that were below it. This iterative process was essentially the same process used in Weierstrass's proof of the maximum value theorem (see [27, p. 953]). In particular, Bolzano's lemma allowed Weierstrass to prove that every bounded infinite set of real numbers has a limit point. It is this property that Fréchet used when he generalized Weierstrass's theorem to abstract spaces. We now know this property as the Bolzano-Weierstrass property, or limit-point compactness.

While Bolzano and Weierstrass were trying to characterize properties of the real line in terms of sequences, other mathematicians, such as Borel and Lebesgue, were trying to characterize it in terms of open covers. Borel proved the following lemma in his 1894 thesis.

Lemma 2.3 (Borel). If on a line one has an infinite number of subintervals, such that every point of the line is interior to at least one of the intervals, then one can determine effectively a bounded number of intervals from among the given intervals that have the same property (every point of the line is interior to at least one of them). ${ }^{5}$

Here a line means a bounded interval. It turns out that Borel's approach was similar to the approach Heine used in 1872 to prove that a continuous function on a closed interval was uniformly continuous [23, p. 188]. This theorem was first proven by Dirichlet in his lectures of 1852, with a more explicit use of coverings and subcoverings than in Heine's theorem [15, p. 91]. However, Dirichlet's notes were not published until 1904, which might explain why he does not get credit for the generalized version of the Borel lemma (now referred to as Borel theorem). The reason that Heine's name is attached to the theorem is that Schönflies, a student of Weierstrass, noticed the connection between Heine's work and Borel's [40, p. 51]. The generalized theorem, which is now commonly called the Heine-Borel theorem ${ }^{6}$, with modern language and notation, is as follows:

Theorem 2.4 (Heine-Borel). A subset of $\mathbb{R}$ is compact if and only if it is closed and bounded.

While Heine is credited with a theorem he did not prove, it appears that Cousin was largely overlooked for a lemma he did prove. In 1895 he generalized the Borel lemma

\footnotetext{
${ }^{4}$ From the original German: Wenn eine Eigenschaft $M$ nicht allen Werthen einer veränderlichen Grösse $x$, wohl aber allen, die kleiner sind, als ein gewisser $u$, zukömmt: so gibt es allemahl eine Grösse $U$, welche die grösste derjenigen ist, von denen behauptet werden kann, dass alle kleineren $x$ die Eigenschaft $M$ besitzen [7, p. 41].

${ }^{5}$ From the original French: Si l'on a sur une droite une infinitè d'intervalles partiels, tels que tout point de la droite soit intérieur à l'un au moins des intervalles, on peut déterminer effectivement un nombre limité d'intervalles choisis parmi les intervalles donnés et ayant la même propriété (tout point de la droite est intérieur à au moins l'un d'eux) [8, p. 51].

${ }^{6}$ For an accessible history of this theorem, along with discussion of a number of different original statements of this theorem, see [2].
} 
to arbitrary covers. In the formulation of the lemma below, the plane YOX is just $\mathbb{R}^{2}$, and the region $S$ would, in today's language, be described as closed and bounded.

Lemma 2.5 (Cousin). In the plane YOX, let $S$ be a connected area bounded by a closed contour, simple or complex. Suppose that at each point of $S$ or its perimeter there is a circle, of nonzero radius, having this point as its center. It is then always possible to subdivide $S$ into regions, finite in number and sufficiently small for each one of them to be entirely inside a circle corresponding to a suitably chosen point in $S$ or on its perimeter. ${ }^{7}$

In other words, if for every point of a closed, bounded region, there corresponds a finite neighborhood, then the region can be divided into a finite number of subregions such that each subregion is contained in a circle having its center in the subregion. ${ }^{8}$ Cousin's lemma (sometimes referred to as Cousin's theorem) is generally attributed to Lebesgue, who was said to be aware of the result in 1898 and published his proof in 1904 [29]. ${ }^{9}$ The Lebesgue lemma is considered itself to be an important consequence of compactness.

While there is some debate over who was really responsible for the ideas and proofs, the idea that any closed, bounded subset of $\mathbb{R}$ has the open-cover property (sometimes called the Borel-Lebesgue property) was known when Fréchet first defined compactness formally.

Spaces of Continuous Functions. A second motivation for the notion of compactness was the study of abstract spaces such as spaces of continuous functions, $C^{0}[a, b] .{ }^{10}$ In $C^{0}[a, b]$, points are functions (whereas in $[a, b]$ points are real numbers). ${ }^{11}$ The properties of $[a, b]$ alone might not have been seen as important to generalize if it weren't the case that these properties seemed to be important in more abstract spaces as well. However, it turns out that infinite dimensional spaces like $C^{0}[a, b]$ are not as well behaved as finite dimensional spaces like $\mathbb{R}^{n}$. For instance, closed, bounded subsets of continuous functions on $\mathbb{R}$ do not necessarily have the Bolzano-Weierstrass or open-cover property. The work in this area was done by Ascoli and Arzelà in the last decades of the 1800 s.

The following example illustrates that a closed, bounded subset of continuous functions on $\mathbb{R}$ is not, in our modern language, sequentially compact. Consider $B$, the set of continuous functions $f$ defined on $[0,1]$ with $\|f\| \leq 1$. (This is the closed unit ball in $C^{0}[0,1]$ and \|\| is the sup norm.) We will show that there is a sequence in $B$ that does not have a convergent subsequence. Let $f_{n}(x)=x^{n}$. This sequence lies in $B$, but we

\footnotetext{
${ }^{7}$ From the original French: Soit, sur le plan YOX, une aire connexe $S$ limitée par un contour fermé simple ou complexe; on suppose qu'à chaque point de $S$ ou de son périmètre correspond un cercle, de rayon non nul, ayant ce point pour centre: il est alors toujours possible de subdivider $S$ en régions, en nombre fini et assez petites pour que chacune d'elles soit complètement intérieure au cercle correspondant à un point convenablement choisi dans $S$ ou sur son périmètre [12, p. 22].

${ }^{8}$ Note that the original definition was formulated without the term "neighborhood," or "voisinage" in French. The idea of neighborhood was obviously around during Cousin's time, but was not used consistently. Formal definitions of the term can be found in [18] and [22].

${ }^{9}$ Cited in [25, p. 29].

${ }^{10}$ For more details see $[\mathbf{1 6}]$ and $[\mathbf{1 6}]$.

${ }^{11}$ We could just as well take $C^{0}$ on any set $\Omega$.
} 
cannot find a subsequence that converges uniformly to a function in $C^{0}[0,1]$. Suppose to the contrary $f$ is such a function. Then

$$
f(x)=\lim _{k \rightarrow \infty} f_{n_{k}}(x),
$$

which would imply that

$$
f(x)= \begin{cases}0 & \text { if } x<1 \\ 1 & \text { if } x=1\end{cases}
$$

Since $f$ is a discontinuous function, it is not in $C^{0}[0,1]$. Hence the sequence $f_{n}(x)$ has no uniformly convergent subsequence.

The problem in this example comes from how the functions converge. If convergence means pointwise convergence, then we get a behavior different from that of, say, sequences in closed unit balls of $\mathbb{R}^{n}$. In order to avoid this problem, Ascoli introduced the notion of equicontinuity [4, p. 566]. ${ }^{12} \mathrm{~A}$ set $E$ is equicontinuous if and only if for all $\epsilon>0$ there exists a $\delta>0$ such that $|s-t|<\delta$ and $f \in E$ imply $|f(s)-f(t)|<\epsilon$.

The Arzelà-Ascoli theorem, in modern language, then states the following.

Theorem 2.6 (Arzelà-Ascoli). Any bounded equicontinuous sequence of functions in $C^{0}[a, b]$ has a uniformly convergent subsequence. ${ }^{13}$

Using modern terminology we can state a consequence of this theorem, analogous to the Heine-Borel theorem.

Theorem 2.7. A subset of $C^{0}[a, b]$ is compact if and only if it is closed, bounded, and equicontinuous.

Ascoli proved the sufficiency of this condition in 1884 [4, p. 567] and Arzelà the necessity in 1889 [3, p. 345] (with a clearer proof presented in 1894 [5, p. 226]). This generalization of Bolzano-Weierstrass's theorem (although not stated in terms of compactness) was apparently well known after 1880 . Moreover, Hilbert seems to have discovered this compactness property independently and published it in 1900 [13, p. 82]. It is unclear whether Arzelà and Ascoli themselves were aware of how their work was connected with compactness, but Fréchet's work was influenced by theirs [43, p. 255].

Solutions to Differential Equations. A third motivation for the notion of compactness came from the desire to find solutions to differential equations. Peano, a contemporary of Arzelà and Ascoli as well as a fellow Italian, realized that the Arzelà-Ascoli theorem might be useful for demonstrating the existence of such solutions. He searched for solutions by making a sequence of approximations. He

\footnotetext{
${ }^{12}$ See also [9, p. 27].

${ }^{13}$ From the original Italian: La condizione necessaria e sufficiente affinché una successione data di funzioni $u_{1}(x), u_{2}(x), \ldots, u_{n}(x), \ldots$ abbia una funzione limite, nel senso detto sopra, é che, preso un numero positivo $\sigma$ piccolo a piacere, si possa sempre determinare un numero intero corrispondente $m_{\sigma}$ tale che per ogni $x$, nell'intervallo $a \cdots b$, si abbia qualunque sia $p$ intero positivo, $\left|u_{m_{\sigma}(x)}-u_{m_{\sigma}+p}(x)\right|<\sigma$. Translated to English: The necessary and sufficient conditions that a given sequence of functions $u_{1}(x), u_{2}(x), \ldots, u_{n}(x), \ldots$ defined on an interval $a \cdots b$ may converge to a limiting function is that, given an arbitrarily small positive number $\sigma$ there can always be determined a corresponding integer $m_{\sigma}$ such that for all values of $x$ in the interval $a \cdots b$, and for all positive integers $p,\left|u_{m_{\sigma}(x)}-u_{m_{\sigma}+p}(x)\right|<\sigma[\mathbf{5}, \mathrm{p}$. 226].
} 
then used what we now call compactness to show that there will be a subsequence that converges uniformly to a limit (which will be the solution to the differential equation).

To this end, Peano proved the following theorem in 1890.

Theorem 2.8 (Peano). Suppose we are given a system of differential equations in normal form:

$$
\begin{aligned}
\frac{d x_{1}}{d t} & =\varphi_{1}\left(t, x_{1}, \ldots, x_{n}\right), \\
\ldots \ldots & \\
\frac{d x_{n}}{d t} & =\varphi_{n}\left(t, x_{1}, \ldots, x_{n}\right),
\end{aligned}
$$

where the functions $\varphi_{1}, \ldots, \varphi_{n}$ are continuous in a neighborhood of $\left(b, a_{1}, \ldots, a_{n}\right)$. Then there exists an interval $\left(b, b^{\prime}\right)$, and $n$ functions of $t$ from this interval, $x_{1}, \ldots, x_{n}$, satisfying our system of equations and evaluating to $a_{1}, \ldots, a_{n}$, respectively, at $t=b^{14}$

While it is not clear if Fréchet was aware of this application, applications for the notion of compactness were apparently known before the term was formally defined.

3. DEVELOPING THE DEFINITION. We will trace below the development of the two central notions of compactness discussed above, those stemming from sequences and open covers of real numbers. Again, it is useful to know something about the climate of the mathematics community at the time of these historical developments. We will focus on the contributions of only a few central people, but there was actually a large community of mathematicians who were developing ideas that are now the foundations for analysis and topology. Many of these mathematicians were in very close contact with each other, so it is difficult to separate their contributions. Among them, in France, were Hadamard, Lebesgue, and Fréchet; in Russia, Alexandroff ${ }^{15}$ and Urysohn ${ }^{16}$; in Germany, Hausdorff, Hilbert, Schönflies, and Cantor; in Hungary, F. Riesz; in the Netherlands, Brouwer; in Austria, Vietoris; and in the U.S., Chittenden, Hedrick, and Moore.

We will start with the work of Fréchet, who coined the term "compact" and gave definitions for what we now know as countable and sequential compactness. We will then briefly discuss contributions by Alexandroff and Urysohn, who developed and stated what we now call open-cover compactness, or simply compactness. We will show why open-cover and sequential compactness are not equivalent in abstract topological spaces, providing motivation for a formulation of compactness in terms of nets and filters which is analogous to sequential compactness.

\footnotetext{
${ }^{14}$ From the original French: Soit donné le système d'équations différentielles, ramené à la forme normale: $\frac{d x_{1}}{d t}=\varphi_{1}\left(t, x_{1}, \ldots, x_{n}\right), \ldots \frac{d x_{n}}{d t}=\varphi_{n}\left(t, x_{1}, \ldots, x_{n}\right)$, où les $\varphi_{1}, \ldots, \varphi_{n}$ sont des fonctions continues aux environs de $t=b, x_{1}=a_{1}, \ldots, x_{n}=a_{n}$. [Alors il existe] un intervalle $\left(b, b^{\prime}\right)$, et, dans cet intervalle, $n$ fonctions $x_{1} \cdots x_{n}$ de $t$, qui satisfont aux équations données, et qui, pour $t=b$, prennent les values $a_{1} \cdots a_{n}[35$, p.182].

${ }^{15}$ I will use the spelling "Alexandroff," rather than the sometimes used "Alexandrov," since that was the spelling he preferred [11, p. 634].

${ }^{16}$ Urysohn died, tragically, at the age of 26 in a swimming accident off the coast of France. Much of his work was published posthumously by Alexandroff, who kept up his correspondence with Fréchet after Urysohn's untimely death $[\mathbf{4 4}$, p. 340].
} 
Fréchet: Countable and Limit-Point Compactness. While Fréchet was influenced by many contemporaries and predecessors, it seems he deserves credit as the father of compactness. It was Fréchet who gave the concept a name, in a paper [17] leading to his 1906 doctoral thesis. Fréchet also defined metric spaces for the first time, though not using that term, ${ }^{17}$ and made inroads into functional analysis, thus providing a context for which the importance of compactness became clear.

Fréchet was a mathematician of big ideas. He preferred definitions that had an intuitive feel rather than analytic power. This preference can be seen in [17, p. 849] in which he defined a notion of compactness, introducing first a definition of what we now call countable compactness, using nested intersections, before introducing a characterization using limit points.

In Fréchet's thesis, he considered three kinds of spaces, which he called L-class, V-class, and E-class. L-classes were the most general, in which a notion of sequential compactness was defined. E-classes, which we now call metric spaces, and V-classes, ${ }^{18}$ a metric space with a weak version of the triangle inequality, were less general, but easier to work with. The goal was to define compactness for L-classes, but this turned out unsuccessful because sequential compactness did not have all the properties needed to generalize to abstract topological spaces (more on this in section 3.2). Fréchet focused instead on the V-and E-classes, in which notions of modern-day compactness and sequential or limit-point compactness were equivalent. The following definition was given for E-classes.

Definition 3.1. A set $E$ is called compact if, whenever $E_{n}$ is a sequence of nonempty, closed subsets of $E$ such that $E_{n+1}$ is a subset of $E_{n}$ for each $n$, then there is at least one element that belongs to all of the $E_{n}$ 's. ${ }^{19}$

The exact nature of Fréchet's intuition for this definition is unclear, but there might be two features of compact sets that he wanted to capture. The first is a sense of boundedness. The nested intersection property allows us to easily rule out sets that have tails running to $\infty$. For instance, we can show that $\mathbb{R}$ is not compact. Let $E_{n}=[n, \infty)$. Each $E_{n}$ is closed because it contains the point $n$, and clearly $E_{n+1} \subset E_{n}$. However, the infinite intersection of these intervals is empty, so $\mathbb{R}$ is not compact.

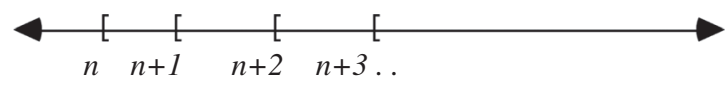

Figure 2. Nested tails

A second feature the nested intersection definition allows us to quickly see is that sets that have "holes" are not compact. For instance, we can see that $X=[a, b]$ is compact and $Y=[a, b) \cup(b, c]$ is not. ${ }^{20}$ In the latter case, consider $E_{i}=\left[a_{i}, b\right) \cup$ $\left(b, c_{i}\right]$, where $a_{i+1}>a_{i}$ and $a_{i} \rightarrow b, c_{i+1}<c_{i}$, and $c_{i} \rightarrow b$. These sets are clearly nested and are closed in $Y$, but the infinite intersection of those intervals is empty. Hence $Y$ is not compact.

\footnotetext{
${ }^{17}$ The term "metric space" is credited to Hausdorff who used the term in [22, p. 290-1].

${ }^{18}$ The letter $\mathrm{V}$ comes from the French voisinage meaning neighborhood, L from limite, or limit, and $\mathrm{E}$ from écart, or "gap," referring to the nonzero distance between two points in a metric space, which according to [20] derived from Jordan's use of the term "distance."

${ }^{19}$ From the original French: $\mathrm{Si}$ on considère une suite d'ensembles $E_{1}, E_{2}, \ldots, E_{n}, \ldots$ formés d'éléments d'un même ensemble compact $E$, chacun fermé, contenu dans le précédent et possédent au moins un élément, il y a nécessairement un élément commun à tous ces ensembles [18, p. 7].

${ }^{20}$ One could construct a similar example with a hole that is compact such as $Z=[a, b] \cup[c, d]$, so the example above only illustrates the intuition.
} 

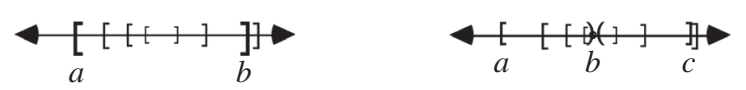

Figure 3. Nested intersections

While Fréchet preferred his intuitive definition involving nested intersections [36, p. 430], he realized the importance of also providing a more useful, if less intuitive, definition. Below is another definition from Fréchet, which uses the BolzanoWeierstrass property. This definition applies to V- and E-classes where limit-point, countable, and sequential compactness are equivalent. Note that for Fréchet, a compact set need not be closed. So for his subsequent definitions and theorems, he often needed to require that a set be both compact and closed.

Definition 3.2. We will say that a set is [relatively limit-point] compact if it contains only a finite number of points or if every one of its infinite subsets gives rise to at least one limit point. ${ }^{21}$

Since Fréchet did not require that a compact set be closed, he defined the notion of an extremal set, which is closer to our modern day notion of compact.

Definition 3.3. We will call a set that is both closed and compact an extremal set. ${ }^{22}$

Fréchet also includes a note which reflects the intuition of this definition, "Within abstract set theory, extremal sets play a role akin to that of intervals in the theory of subsets of the real line." 23 We might not know exactly why Fréchet chose the word "compact," but his choice of the term was not popular with all mathematicians. For instance, Schönflies suggested that what Fréchet called compact be called something like "lückenlos" (without gaps, closer to the modern notion of completeness) or "abschliessbar" (closable), [43, p. 266], suggesting that the intuition behind the term was, at this point, not fully shared. Despite all of Fréchet's early concern with intuitive definitions and choice of terminology, at the end of his life, he could not remember why he chose the term:

Doubtless I wanted to avoid a solid dense core with a single thread going off to infinity being called compact. This is a hypothesis because I have completely forgotten the reasons for my choice! ${ }^{24}$

So even in the lifetime of the mathematician who named the concept, the original intuition behind the concept was somewhat lost, and Fréchet's intuitive nested intersection definition was supplanted by less intuitive but more powerful notions of limit-point, sequential, and open-cover compactness.

Hausdorff: Compactness on Metric Spaces. One of the obstacles to defining compactness, as we know it today, was to define it in a way that would work for abstract

\footnotetext{
${ }^{21}$ From the original French: Nous dirons qu'un ensemble est compact lorsqu'il ne comprend qu'un nombre fini d'éléments ou lorsque toute infinité de ses éléments donne lieu à au moins un élément limite [18, p. 6].

${ }^{22}$ From the original French: Lorsqu'un ensemble est à la fois compact et fermé nous l'appellerons ensemble extrémal [18, p. 6-7].

${ }^{23}$ From the original French: Le rôle de l'ensemble extrémal dans la théorie des ensembles linéaires. [18, p. 7].

${ }^{24}$ From the original French: ... j'ai voulu sans doute éviter qu'on puisse appeler compact un noyau solide dense qui n'est agrémenté que d'un fil allant jusqu'à l'infini. C'est une supposition car j'ai complètement oublié les raisons de mon choix! [36, p. 440].
} 
topological spaces. This was a problem for Fréchet, and in the end he had to restrict his definition to $\mathrm{V}$ - and E-classes, leaving open the question of defining compactness for L-classes, the predecessor of what we now call abstract topological spaces. In the early 1900s, Hausdorff's work revolutionized the area of topology, providing definitions that are now standard in the field. ${ }^{25}$ For instance, in 1914 he introduced what we now call Hausdorff spaces, in which distinct points have disjoint neighborhoods. In [22] he defined a set $E$ to be compact if every infinite subset of $E$ has a limit point in $E$, where limit point in this context means that every neighborhood of the point contains infinitely many elements of $E$. Hausdorff's notion of compactness, which we would call limit-point compactness and is equivalent to countable compactness for Hausdorff spaces, remained the standard notion of compactness throughout the rapid development of point-set topology in the 1920 s. $^{26}$

Alexandroff and Urysohn: Open-Cover Compactness. While Fréchet was the first to formally define compactness, his contemporaries in Russia, Alexandroff and Urysohn, appear to be the first to state it in its most general form, in the context of abstract topological spaces. It is perhaps for this reason that the two Russians are often credited with defining the notion (e.g., [30, p. 425S]).

In a paper in 1923, Alexandroff and Urysohn listed open-cover compactness, the property that every open cover has a finite subcover, as one of three equivalent properties a set could have to be called compact (in their language "bicompact"). The other two properties were that all infinite sets have a complete accumulation point and that nested intersections are nonempty. ${ }^{27}$ Alexandroff and Urysohn note that these three properties were already known, although the concept had not been named. ${ }^{28}$ Alexandroff claimed the accumulation point characterization was most important initially, due to the dominance of the Bolzano-Weierstrass property, but after some years it became clear that the open-cover property was more fruitful [11, p. 633]. Today it is common to give the open-cover property as the definition and show the equivalence of one or both of the other two properties as theorems. ${ }^{29}$ While more abstract, and perhaps less intuitive than the other characterizations, the open-cover property brings out more clearly than the other ones the analogy between compactness and finiteness. ${ }^{30}$

Alexandroff and Urysohn were actually in close contact with Fréchet [44, pp. 319357] during the time they developed their work on compact topological spaces. Although Alexandroff and Urysohn usually get credit for defining open-cover compactness, Fréchet was not unaware of the possibility of using neighborhoods to characterize

\footnotetext{
${ }^{25}$ See [37] for more details and a biographical account of the life of this intellectual giant, including a parallel career under the pseudonym Paul Mongré, and his eventual suicide in the internment camp Bonn-Endenich in 1942.

${ }^{26}$ Thanks to an anonymous reviewer for this information and formulation. See [28] for more detail about Hausdorff's role in the development of topology and related fields.

${ }^{27}$ From the original French: En appelant point d'accumulation complète d'un ensemble $E$ situé dans l'espace topologique $R$ tout point $\xi$ tel que la puissance de la partie de $E$ contenue dans un voisinage quelconque du point $\xi$ est égale à celle de tout l'ensemble $E$-les trois propriétés suivantes sont équivalentes: (A) Tout ensemble infini situé dans $R$ possède un au moins point d'accumulation complète, (B) Toute suite bien ordonnée descendante d'ensembles fermés situés dans $R$ possède au moins un point commun à tous les ensembles de la suite, (C) De toute infinité de domaines recouvrant l'espace $R$ on peut extraire un nombre fini jouissant de la même propriété $[\mathbf{1}, \mathrm{p} .6]$.

${ }^{28}$ Sans prétendre de donner une notion nouvelle, nous allons appeler bicompacts les espaces $R$ vérifiant une quelconque (et, par suite, toute les trois) des propriétés énoncées [1, p. 6].

${ }^{29}$ An early example of this is [26, p. 135-137].

${ }^{30}$ Reminiscent of the joke, attributed to Herman Weyl [24, p. 499], "What is a compact city? It is one that can be guarded by finitely many near-sighted policemen."
} 
compactness, an idea suggested by his advisor Hadamard in $1905 .^{31}$ The first definition that Fréchet gave, in terms of nested intersections, is the dual of, and hence logically equivalent to, countable open-cover compactness.

Open-Cover vs Limit-Point Compactness. Although Fréchet may have been motivated originally to define compactness for abstract topological spaces, he in fact restricted himself to metric spaces. His approach of looking at sequences and limits was not as general as the approach of using open covers, which resulted in what we now take to be the correct definition of compactness. ${ }^{32}$ Here we look at examples which illustrate why sequential compactness and open-cover compactness are not equivalent. We will use the concept of the least upper bound property, namely that any nonempty set containing an upper bound necessarily has a least upper bound.

Sequentially Compact Does Not Imply Compact. Consider $S_{\Omega}=\{\alpha \mid \alpha$ is an ordinal number and $\alpha<\Omega\}$ with the order topology, where $\Omega$ is the first uncountable ordinal number. ${ }^{33}$ See Figure 4 below. The first infinite ordinal, $\omega$, is the first ordinal after "exhausting" the natural numbers. The first uncountable ordinal, $\Omega$, is the ordinal after "exhausting" the countable ordinals.

We know that all closed subsets of compact sets are compact (and all compact sets

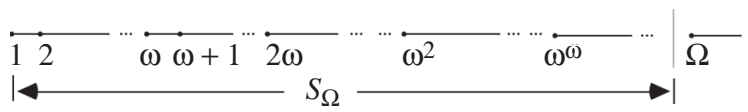

Figure 4. Representation of $S_{\Omega}$

are closed). So $S_{\Omega}$ is not compact since it is not closed in the compact set $S_{\Omega} \cup\{\Omega\}$. However, it turns out that $S_{\Omega}$ is limit-point compact. To see why this is true, we will use the fact that any countable subset of $S_{\Omega}$ has an upper bound in $S_{\Omega}$. If we take any infinite subset of $S_{\Omega}$, it has a countably infinite subset, which we will call $X$. Since $X$ is countable, it has an upper bound, let's call it $b$, in $S_{\Omega}$. But the interval $[1, b]$ is compact since $S_{\Omega}$ has the l.u.b. property. So there must be a point in $[1, b]$ which is a limit point (of both $X$ and any set containing it). Thus, $S_{\Omega}$ is limit-point compact. Essentially the same argument shows that any sequence in $S_{\Omega}$ must have a convergent subsequence in $S_{\Omega}$, so $S_{\Omega}$ is sequentially compact.

Compact Does Not Imply Sequentially Compact. Just as we can have a space that is compact but not sequentially compact, we can also have a space that is sequentially compact but not compact. ${ }^{34}$ Consider the set of all functions from the interval $[0,1]$ to itself with the topology of pointwise convergence. This can be thought of as the infinite product $[0,1]^{[0,1]}$ with the product topology, which is compact by Tychonoff's theorem. ${ }^{35}$ However, if $f_{n}(x)$ is the $n$th digit in the base- 2 decimal expansion of $x$

\footnotetext{
${ }^{31}$ See [28, p. 212]. The undated letter from Hadamard to Fréchet and can be found in full in [43, p. 246].

${ }^{32}$ For more detail on how the open-cover definition became standard, see [11, p. 633-635]. An interesting anecdote attributed to Melvin Henrikson is included in this paper concerning the emotions behind the establishment of the now standard topological definitions. An attempt was made in 1925 in Ann Arbor to nail down the definitions, but after an intense hour long debate, the meeting broke up —mostly over disagreement on how to define compactness!

${ }^{33}$ Refer to [42, p. 68] for further discussion of this example.

${ }^{34}$ For more detail see [42, p. 125-126].

${ }^{35}$ The product of compact topological spaces is compact, or in German, Das Produkt von bikompakten Räumen ist wieder bikompakt, originally proved in [45, p. 772], though the theorem is sometimes credited to Čech [16].
} 
(using the expansion that terminates in 0's if $x$ is a dyadic rational), the sequence $f_{n}$, which is a sequence in the set of all functions from $[0,1]$ to itself, has no pointwise convergent subsequence. It does have convergent subnets,${ }^{36}$ a concept that will be defined in the next section, but not proper convergent sequences.

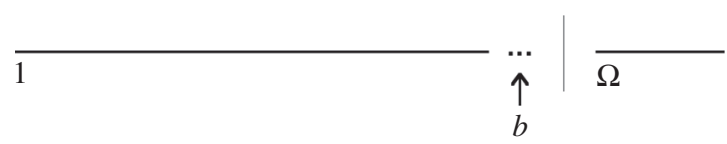

Figure 5. Illustration of $b$ in $S_{\Omega}$.

4. NETS AND FILTERS. In the previous section we saw that the two important properties of compactness, those stemming from the Bolzano-Weierstrass property (sequential compactness) and the Borel-Lebesgue property (open-cover compactness), are not equivalent in abstract topological spaces. Open-cover compactness is more general and applicable and for these reasons is now the concept to which the term "compactness" refers. However, it is possible to define open-cover compactness in a way that is analogous to sequential compactness, using notions of nets and filters. ${ }^{37}$ These two concepts are very different on the surface, but they give rise to the same notion of convergence in abstract topological spaces.

Moore and Smith: Nets. The theory of nets was developed by E. H. Moore and his student H. L. Smith, and published in 1922 [32]. ${ }^{38}$ It is unclear whether Moore and Smith knew how nets could be used to define compactness. This connection is usually credited to Birkhoff [26, p. 64], who applied Moore-Smith theory to general topological spaces. However, in the paper in which Moore and Smith introduce the concept of nets, they also generalize some of Fréchet's compactness results [32, p. 118]. Our goal here is to express compactness in terms of nets, and we will use the $S_{\Omega}$ example to motivate and illustrate net compactness.

The problem in the $S_{\Omega}$ example is that while $\Omega$ is a limit point of $S_{\Omega}$ (any neighborhood of $\Omega$ contains points of $S_{\Omega}$ ), no sequence in $S_{\Omega}$ converges to $\Omega$ [26, p. 76]. If we are limited to taking a countable number of elements in the sequence, we will never reach $\Omega$. Nets provide one way of getting around this problem by allowing us to have something like uncountable sequences. In our discussion of both nets and filters, we will consider only topological spaces, on which the notion of neighborhood is defined. ${ }^{39}$

To see how nets are a generalization of sequences, it is useful to think of sequences as functions on the natural numbers.

Definition 4.1. A sequence (denoted $\left\{x_{n}\right\}_{n \in \mathbb{N}}=\left\{x_{1}, x_{2}, x_{3}, \ldots\right\}$ ) is a function which assigns to each element $n$ of the natural numbers, $\mathbb{N}$, a functional value $x_{n}$ in a set $X$.

\footnotetext{
${ }^{36}$ Every function from $[0,1]$ to $\{0,1\}$ is the limit of some subnet.

${ }^{37}$ In this section we are more interested in the concepts and not the historical development, so we will be less careful than in earlier sections about giving original definitions and theorems, though we provide references for anyone who wants to track down the original formulations.

${ }^{38}$ Little biographical information about Smith is available. He received his Ph.D. from University of Chicago under Moore and got a job at Louisiana State University, but apparently after his important work on nets and filters, he dropped into obscurity [19, p. 563].

${ }^{39}$ This treatment follows [26, pp. 62-70] and [31, pp. 281-283, 286-289].
} 
We would like to replace $\mathbb{N}$ with a set that can be uncountable but has an ordering similar to that of $\mathbb{N}$. In other words, we want to stipulate conditions for an ordering relation on a generic set that generalizes the way $>$ orders natural numbers. We will call this relation $\succ$ to suggest the connection to $>$, and we will say that this relation "directs" a given set.

Definition 4.2. A nonempty set $D$, with the relation $\succ$ is called directed if and only if

(i) if $d_{1}, d_{2}, d_{3} \in D$ such that $d_{1} \succ d_{2}$ and $d_{2} \succ d_{3}$, then $d_{1} \succ d_{3}$;

(ii) if $d_{1}, d_{2} \in D$, then there is a $d_{3} \in D$ such that $d_{3} \succ d_{1}$ and $d_{3} \succ d_{2}$.

So the definition of a net is simply the definition of a sequence with $\mathbb{N}$ replaced by the notion of a directed set. From now on, $D$ will stand for a directed set with the relation $\succ$ as defined above.

Definition 4.3. A net (denoted $\left\{x_{d}\right\}_{d \in D}$ or simply $\left\{x_{d}\right\}$ ) is a function which assigns to each element $d$ of a directed set $D$ a functional value $x_{d}$ in a set $X$.

Once we know what a net is, we can state what it means for it to converge. Again we can derive the definition for net convergence and limit point by taking the definitions involving sequences and simply replacing $\mathbb{N}$ and $>$ with $D$ and $\succ$.

Definition 4.4. A net $\left\{x_{d}\right\}$ converges to $a \in X$ (denoted $\left\{x_{d}\right\} \rightarrow a$ ) if and only if for every neighborhood $U$ of $a$, there is an index $d_{0} \in D$ such that if $d \succ d_{0}$, then $x_{d} \in U$ (i.e.. if the net is eventually in each neighborhood of $a$ ).

Definition 4.5. A point $a$ is a limit point of $\left\{x_{d}\right\}$ if for every neighborhood $U$ of $a$ and every $d_{0} \in D$, then there is a $d \succ d_{0}$ such that $x_{d} \in U$.

In order to state compactness in terms of nets, we also need the concept of subnet, the analog of subsequence. ${ }^{40}$ Part of the definition of subsequence generalizes easily, but the other part requires us to think about subsequences in a slightly different way than we are accustomed. The first defining property of subsequence is that each element of the subsequence can be identified with an element of the sequence. This property is generalized in (i) below. The second defining property requires that the subsequence is ordered in a similar way as the sequence. Usually we require the indices of the subsequence, like the indices of the sequence, to be strictly increasing. In other words, for a subsequence $\left\{x_{n_{k}}\right\}$ of a sequence $\left\{x_{n}\right\}$, the $n_{k}$ are positive integers such that $n_{1}<n_{2}<n_{3} \cdots$. But the feature of this condition which turns out to be important is simply the fact that as $k \rightarrow \infty$, so do the $n_{k}$. This property is generalized in (ii) below.

Definition 4.6. A subnet of a net $\left\{x_{d}\right\}_{d \in D}$ is a net $\left\{y_{b}\right\}_{b \in B}$ where $B$ is a directed set and there is a function $\varphi: B \rightarrow D$ such that:

(i) $y_{b}=x_{\varphi(b)}$ and

(ii) $\forall d \in D, \exists b_{0} \in B$ such that if $b \succ b_{0}$, then $\varphi(b) \succ d$.

We are now ready to characterize compactness in terms of nets.

\footnotetext{
${ }^{40}$ Incidentally, Kelley, who first coined the term "net" had considered using the term "way" so the analog of subsequence would be "subway." McShane also proposed the term "stream" for net since he thought it was intuitive to think of the relation of the directed set as "being downstream from" [31, p. 282].
} 
Theorem 4.7. A topological space $X$ is compact if and only if either,

(i) every net of points of $X$ has a limit point in $X$, or

(ii) every net of points of $X$ has a convergent subnet in $X$.

Notice that these definitions are precisely the same as limit point and sequential compactness for metric spaces with the term "net" substituted for "sequence."

Applying these definitions to the $S_{\Omega}$ example, we can show why $S_{\Omega}$ is not compact. If we take a net $\left\{x_{d}\right\}$ of elements of $S_{\Omega}$, it is no longer the case that there will necessarily be a limit in $S_{\Omega}$. In particular, let $D=S_{\Omega}$ and $x_{d}=d$. Then $\left\{x_{d}\right\}$ converges to $\Omega$, which is not in $S_{\Omega}$. Thus no subnet of $\left\{x_{d}\right\}$ will converge to a point in $S_{\Omega}$.

Cartan (and Smith): Filters. Nets are not the only way of generalizing sequences. Another generalization of sequence is a filter, defined by H. Cartan in $1937 .{ }^{41}$ While different from a net, both nets and filters give rise to the same notion of convergence on topological spaces. That is to say, on abstract topological spaces, they are essentially the same. ${ }^{42}$ Nonetheless, some mathematicians find nets more intuitively appealing and useful, while others prefer filters.

The idea behind filters was foreshadowed by F. Riesz in 1907 when he provided axioms for topology based on limit points instead of a metric [39]. We note that though his topological axioms are not equivalent to the standard ones we use today, and his work did not result in a fruitful line of research. Riesz defines a concept called an "ideal" which is essentially the same as what we now call an ultrafilter. Smith independently discovered filters as an attempt to explain what was lacking in the theory of nets that he and Moore proposed.

Following our treatment of nets, we will now define the notions we need to state compactness in terms of filters and then apply our compactness result to show $S_{\Omega}$ is not compact. As with nets, we can look at convergence of sequences to motivate the idea of convergence of filters. However, whereas with nets the focus was on the index set, with filters the focus is on neighborhoods. ${ }^{43}$

Definition 4.8. Let $X$ be a set. A set $\Phi$ of subsets of $X$ is called a filter if and only if

(i) $\emptyset \notin \Phi$,

(ii) $A_{1} \subset A_{2} \subset X$ and $A_{1} \in \Phi \Rightarrow A_{2} \in \Phi$, and

(iii) $A_{1}, A_{2} \in \Phi \Rightarrow A_{1} \cap A_{2} \in \Phi$.

As with nets, we should define what it means for a filter to converge.

Definition 4.9. A filter $\Phi$ converges to $a \in A$ (denoted $\Phi \rightarrow a$ ) if and only if each neighborhood of $a$ is a member of $\Phi$.

There is a natural way to associate a filter with any sequence. If $x_{1}, x_{2}, x_{3}, \ldots$ is a sequence in $X$, we can associate with this sequence a filter $\Phi$ on $X$ such that $\forall a \in$ $X,\left\{x_{n}\right\} \rightarrow a$ if and only if $\Phi \rightarrow a$. In particular, let $\Phi=\left\{A \subset X \mid \exists k_{A}\right.$ such that $\left.\forall i \geq k_{A}, x_{i} \in A\right\}$. So the tails of the sequence are contained in neighborhoods which

\footnotetext{
${ }^{41}$ See also $[\mathbf{1 0}$, p. 8$]$.

${ }^{42}$ To show equivalence on abstract topological spaces, there is for example an exercise in Kelley that establishes a dictionary mapping between them (i.e., given a net you can find a filter, and vice versa) [26, p. 83]. But there is a subtle distinction for a particular type of limit found in the advanced theory of integration [41, p. 371].

${ }^{43}$ This treatment follows [14].
} 
are members of the filter. The condition that each neighborhood of $a$ is in the filter is then equivalent to the condition that the sequence is eventually in any neighborhood of $a$.

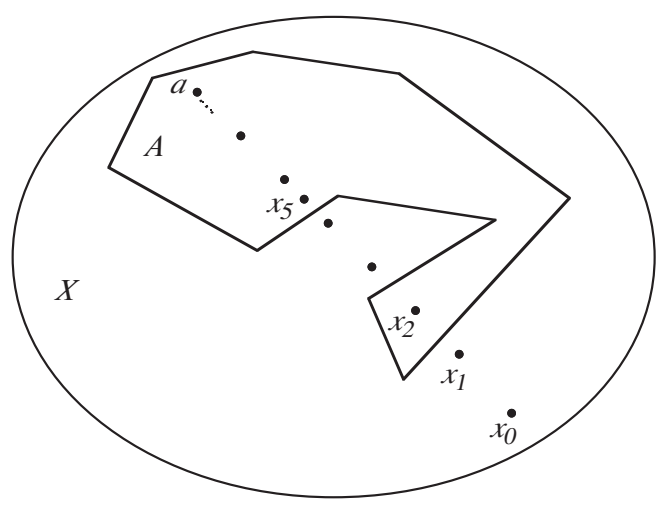

Example with $k_{\mathrm{A}}=5$

Figure 6. Member of filter with $k_{A}=5$

In order to define compactness in terms of filters, we need one more notion, that of an ultrafilter.

Definition 4.10. A filter in $X$ is an ultrafilter if and only if no filter in $X$ properly contains it.

The notion of ultrafilter is not exactly analogous to subsequence, but in the formulation of compactness, it serves the same purpose.

Theorem 4.11. A topological space is compact if and only if every ultrafilter on $X$ converges to a point in $X$.

Now we can return again to our example and get a sense in terms of filters for why $S_{\Omega}$ is not compact. ${ }^{44}$ We want to show that there is an ultrafilter on $S_{\Omega}$ that does not converge. Consider all the neighborhoods of $\Omega$ in $S_{\Omega} \cup \Omega$. Let $\Phi=\left\{A \subset S_{\Omega} \mid \exists \alpha \in S_{\Omega}\right.$ such that $\forall \beta \geq \alpha, \beta \in A\}$.

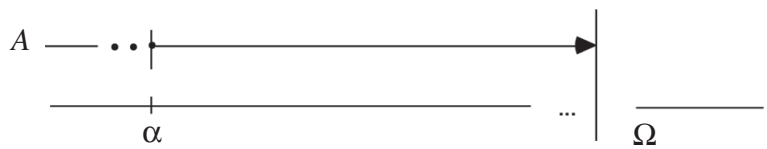

Figure 7. Member of filter on $S_{\Omega}$

This clearly satisfies the definition of a filter. Let $\Psi$ be any ultrafilter containing $\Phi$. We claim $\Psi$ does not converge in $S_{\Omega}$. Suppose it did. Say that $\Psi \rightarrow b$. Now pick some $\alpha>b$ with $\alpha \in S_{\Omega}$. Then $A^{+}=\{\beta: \beta \geq \alpha\} \in \Psi$ since $A^{+} \in \Phi \subseteq \Psi$. We also have $A^{-}=\{\beta: \beta<\alpha\} \in \Psi$ since $A^{-}$is an open neighborhood of $b$ (and we claim $\Psi$ converges to $b$ ).

But $A^{+} \cap A^{-}=\emptyset$, which violates the definition of a filter, so our assumption must be wrong. Thus, $\Psi$ must not converge, and hence $S_{\Omega}$ is not compact.

\footnotetext{
${ }^{44}$ The proof of this claim, in particular when we assert that there is an ultrafilter containing our filter, actually relies on the axiom of choice.
} 


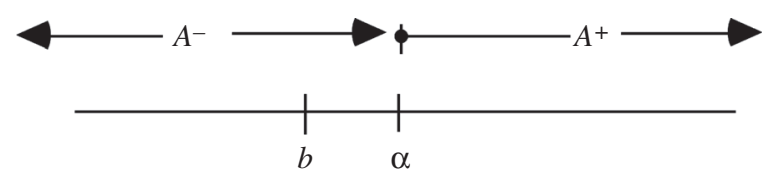

Figure 8. Illustration of $A^{-}$and $A^{+}$

Final Comments. Here ends the story, a sort of co-evolution of the two different but related notions of sequential and open-cover compactness. Today when we use the term "compact" we mean open-cover compact, but this paper, and the terms listed in the Appendix, show that this was not always the case. The story of how open-cover compactness came to be seen as the right one is a story of developing mathematics without always knowing where it was going, how important terms should be defined, and how widely they might be applied.

It might be worth noting, in closing, that even this paper, which attempts to characterize the evolution of compactness, is only a sort of snapshot. In the time it took to write this paper and revise it, textbooks have changed. For instance, the latest version of a standard general topology textbook [34] now includes a discussion of nets and filters, whereas the earlier version [33], available during the writing of the original version of this paper, did not.

The lesson to be drawn is simply that mathematics evolves and changes as concepts become clearer and are applied in more general situations. This may be obvious to the mathematician who is involved in making these conceptual advances, but may be less clear to the student who sees a textbook as a collection of established facts. Textbooks are, as perhaps they should be, a distillation of what we currently know. They are also historical documents in their own right. Being aware of this fact may help students mature as mathematicians.

ACKNOWLEDGMENT. Many thanks to those who have supported this project in ways both small and substantial: Hendrik Lenstra, Hans Wallin, Johan de Jong, Lucien Le Cam, Jeremy Gray, Umberto Bottazzini, Gerald Folland, Michael Saks, Klas Markström, Victor Falgas-Ravry, Edouard Servan-Schreiber, Sophie Laplante, and Lars-Daniel Öhman. Without this help this paper would not have been half as good, and I would not have learned half as much. I am also indebted to three anonymous reviewers for their careful and meticulous feedback. And I am grateful to several librarians, including Mikael Rågstedt at the Mittag-Leffler Institute, who helped me track down original sources. This project was funded in part by a stipend from Wenner-Gren Foundation. All mistakes are, of course, my own.

\section{REFERENCES}

1. P. Alexandroff, P. Urysohn, Sur les espaces topologiques compacts, Bull. Int. Acad. Pol. Sci. Lett. Ser. A (1923) 5-8.

2. N. Andre, S. Engdahl, A. Parker, An analysis of the first proofs of the Heine-Borel theorem - Borel's proof, Loci (2013), http://dx.doi.org/10.4169/loci003890.

3. C. Arzelà, Funzioni di linee, Att. Real. Acca. Lin. 4 no. 5 (1889) 342-348.

4. G. Ascoli, Le curve limite di una varietà data di curve, Att. Real. Acca. Lin. 3 no. 18 (1883/4) 521-586.

5. G. Ascoli, C. Arzelà, Sulle funzioni di linee, Mem. R. Acad. Sci. Inst. Bologna 5 (1894) 225-244, https://archive.org/stream/memoriedellar55189596racc\#page/224/mode/2up.

6. G. Birkhoff, E. Kreyszig, The establishment of functional analysis, Historia Math. 11 (1984) 258-321.

7. B. Bolzano, Rein analytischer Beweis des Lehrsatzes dass zwischen je zwey Werthen, die ein entgegengesetztes Resultat gewähren, wenigstens eine reelle Wurzel der Gleichung liege, Prague, 1817.

8. E. Borel, Sur Quelques Points de la Théorie des Fonctions. Gauthier-Villars, Paris, 1894.

9. N. Bourbaki, Topologie Générale. Book X, Hermann, Paris, 1949.

10. - Topologie Générale. Book XVI, Hermann, Paris, 1953. 
11. R. Chandler, G. Faulkner, Hausdorff compactifications: A retrospective, in Handbook of the History of General Topology. Edited by C. E. Aull, R. Lowen. Springer-Verlag, Berlin, 2001, http://dx. doi . org/10.1007/978-94-017-0468-7.

12. P. Cousin, Sur les fonctions de $n$ variables complexes, Acta Math. 19 (1895) 1-61.

13. J. Dieudonné, History of Functional Analysis. Elsevier Science, Paris, 1981.

14. J. Dixmier, General Topology. Springer-Verlag, New York, 1985, http://dx.doi.org/10.1007/ 978-1-4757-4032-5.

15. P. Dugac, Sur la correspondance de Borel et le theoreme de Dirichlet-Heine-Weierstrass-BorelSchoenflies-Lebesgue, Arch. Inter. Hist. Sci. 39 no. 122 (1989) 69-110.

16. G. Folland, A tale of topology, Amer. Math. Monthly 117 no. 8 (2010) 663-672.

17. M. Fréchet, Généralisation d'un théorème de Weierstrass, C. R. Acad. Sci. Paris 139 (1904) 848-850.

18. - Sur quelques point du calcul fonctionnel, Rend. Palermo 22 (1906) 1-74.

19. P. R. Halmos, Has progress in mathematics slowed down? Amer. Math. Monthly 97 no. 7 (1990) 561-588.

20. Handbook of the History of General Topology. Ed. by C. E. Aull, R. Lowen. Springer-Verlag, Berlin, 2001, http://dx.doi.org/10.1007/978-94-017-0468-7.

21. R. Hartshorne, Algebraic Geometry. Springer-Verlag, New York, 1977, http://dx.doi.org/10. 1007/978-1-4757-3849-0.

22. F. Hausdorff, Grundzüge der Mengenlehre. Verlag von Veit, Leipzig, 1914.

23. E. Heine, Die Elemente der Funktionenlehre, J. Reine Angew. Math. (1872) 172-188.

24. E. Hewitt, The role of compactness in analysis, Amer. Math. Monthly 67 no. 6 (1960) 499-516.

25. T. H. Hildebrandt, The Borel theorem and its generalizations, in The Chauvenet Papers: A Collection of Prize-Winning Expository Papers in Mathematics. Ed. by J. C. Abbott. Mathematical Association of America, Washington, DC, 1925.

26. J. Kelley, General Topology. D. Van Nostrand, Princeton, 1955.

27. M. Kline, Mathematical Thought: From Ancient to Modern Times. Oxford Univ. Press, Oxford, 1972.

28. T. Koetsier, J. Van Mill, By their fruits ye shall know them: Some remarks on the interaction of general topology with other areas of mathematics, in History of Topology. North-Holland Publishing, Amsterdam, 1999. 199-239.

29. H. Lebesgue, Leçons sur l'Intégration et la Recherche des Fonctions Primitives. Gauthier-Villars, Paris, 1902.

30. Mathematical Society of Japan, Encyclopedic Dictionary of Mathematics. Second edition. MIT Press, Cambridge, MA, 1987.

31. E. J. McShane, Partial orderings and Moore-Smith limits, in The Chauvenet Papers: A Collection of Prize-Winning Expository Papers in Mathematics. Ed. by C. Abbott. Mathematical Association of America, Washington, DC, 1950.

32. E. H. Moore, H. L. Smith, A general theory of limits, Amer. J. Math. 44 (1922) 102-121.

33. J. R. Munkres, Topology: A First Course. First Edition. Prentice-Hall, Englewood Cliffs, NJ, 1975.

34. - Topology: A First Course. Second Edition. Prentice-Hall, Englewood Cliffs, NJ, 2000.

35. G. Peano, Démonstration de l'intégrabilité de équations différentielles ordinaires, Math. Ann. 37 (1890) 182-228.

36. J. P. Pier, Historique de la notion de compacité, Historia Math. 7 (1980) 425-443.

37. W. Purkert, The double life of Felix Hausdorff/Paul Mongré. Math. Intelligencer, Trans. by H. and D. Rowe, 30 no. 4 (2008) 36-50.

38. M. Raman, Understanding Compactness: A Historical Perspective, Master of Arts Thesis, Univ. of California, Berkeley, 1997.

39. F. Riesz, Die genesis des Raumbegriffes, Math. Natwiss. 24 (1907) 309-353.

40. A. Schönflies, Die Entwickelung der Lehre von den Punktmannigfaltigkeiten, in Jahresbericht Deutsh Mathers Verein. B.G. Teubner, Leipzig, 1900.

41. H. L. Smith, A general theory of limits, Natl. Math. Mag. 7 no. 8 (1938) 371-379.

42. L. Steen, J. A. Seebach, Counterexamples in Topology. Holt, Rinehart and Winston, New York, 1970, http://dx.doi.org/10.1007/978-1-4612-6290-9.

43. A. Taylor, A study of Maurice Fréchet: I. His early work on point set theory and the theory of functionals, Arch. Hist. Exact Sci. 27 no. 3 (1982) 233-295.

44. — A study of Maurice Fréchet: II. Mainly about his work on general topology 1909-1928, Arch. Hist. Exact Sci. 34 no. 3 (1985) 279-380.

45. A. Tychonoff, Ein Fixpunktsatz, Math. Ann. 111 no. 1 (1935) 767-776.

46. K. Weierstrass, Einleitung in die Theorie der Analytischen Funktionen. Vorlesung Berlin, written down by A. Hurwitz, worked out by P. Ullrich, 1878.

MANYA RAMAN-SUNDSTRÖM is an Associate Professor of mathematics education. She works mostly on the teaching and learning of mathematical proof and on mathematical aesthetics. 
Appendix: Terminology There are many notions related to (but not necessarily equivalent to) compactness. Table 1 contains a list of some of these notions. For a more comprehensive list see [42].

Table 1. Flavors of compactness

[open-cover] compact: Every open cover has a finite subcover.
(also called the Borel-Lebesgue property)
sequentially compact: Every sequence has a convergent subsequence.
countably compact: Every countable open cover has a finite subcover.
limit-point compact: Every infinite subset of $X$ has a limit point in $X$.
(also called Fréchet compact or the Bolzano-Weierstrass property)
relatively compact: The closure is compact.
pseudo-compact: Each continuous real valued function on $X$ is bounded.
finally compact: Every open cover has a countable subcover.
(also called Lindelöf compact)

Many of these concepts are related. For instance, compactness implies countable compactness implies limit point compactness. Sequential compactness implies countable compactness. If we put further restrictions on our spaces we can get implications in the other direction. In $T_{1}$ spaces, limit-point compactness implies countable compactness. In first countable spaces, countable compactness implies sequential compactness. In second countable spaces, sequential compactness implies compactness. In particular, we know that in compact metric spaces, which turn out to be second countable, the first four notions of compactness in Table 1 are equivalent.

It took some time as compactness was applied to different types of spaces for relationships like these to be worked out. It also took time for names to stabilize. Table 2 lists different terms used for compactness-related ideas used by some of the most influential mathematicians in the historical development. In this paper, the modern names have been used.

Table 2. Names of historical compactness-related terms

\begin{tabular}{|l|l|l|l|}
\hline Who & When & Their term & Modern term \\
\hline Fréchet & \multirow{2}{*}{1906} & compact & relatively sequentially compact \\
\cline { 3 - 4 } & & extremal & sequentially compact \\
\hline \multirow{2}{*}{$\begin{array}{l}\text { Russian School } \\
\text { (Alexandroff, etc.) }\end{array}$} & $1920 \mathrm{~s}$ & bicompact & compact \\
\cline { 3 - 4 } & & compact & countably compact \\
\hline Bourbaki & \multirow{2}{*}{1930 s } & quasi-compact & compact \\
\cline { 3 - 4 } & & compact & compact and Hausdorff \\
\hline
\end{tabular}

